\title{
The molecular determination of emm genotypes in non-group a beta-hemolytic streptococci isolated from clinical samples
}

\author{
Suna Kızılyıldırım ${ }^{1}$, Cansu Önlen Güneri² ${ }^{2}$ Fatih Köksal ${ }^{3}$ \\ ${ }^{1}$ Department of Pharmaceutical Microbiology, Faculty of Pharmacy, University of Süleyman Demirel, Isparta, Turkey \\ ${ }^{2}$ Department of Medical Microbiology, Gulhane Vocational School of Health Services, University of Sağlk Bilimleri, Ankara, Turkey \\ ${ }^{3}$ Department of Medical Microbiology, Medical Faculty, Cukurova University, Adana, Turkey
}

Received: 2021-04-13.

Accepted: 2021-06-10

This work is licensed under a

Creative Commons Attribution 4.0 International License

J Clin Med Kaz 2021; 18(4):64-68

Corresponding author:

Suna Kızılyıldırım.

E-mail: suna_ky@hotmail.com,

sunakizilyildirim@sdu.edu.tr;

ORCID: 0000-0002-1039-8556

\section{Abstract}

Objective: $M$ protein is an important marker in epidemiological and phylogenetic surveillance of Beta-Hemolytic Streptococci.

The aim of this study is to determine emm types and their distribution among the Group C/G streptococci (GCG/GGS) strains isolated from clinical samples.

Material and methods: The study includes $98 \beta$-hemolytic streptococcus strains isolated from clinical samples in Çukurova University/Balcalı Hospital. 67 of these isolates, defined as serologic, of which 56 were identified as Streptococcus dysgalactiae subsp. equisimilis, 8 as S. anginosus and 3 as S.equi subsp.zooepedimicus, have been included in the study. These strains were also confirmed by the Vitek 2GP-ID system. These isolates were confirmed by the polymerase chain reaction (PCR) method with primers based on groESL sequences. PCR-sequence analysis method developed by the Centers for Disease Control (CDC) was used for typing according to emm polymorphism.

Results: $56 / 67$ (83.6\%) isolates in which emm-PCR was determined as positive were typed by sequence analysis. The findings were identified using the CDC database, and the most common type was determined to be stG485 (51.85\%), stG643 (12.96\%) and stG6 (9.25\%).

Conclusion: Consequently, GCS/GCS should be treated more seriously in our country as in the whole world.

Key words: emm, PCR, GCS, GGS

\section{Introduction}

Beta hemolytic streptococci (BHS) are often the commensal flora members of various body regions such as upper respiratory tracts, gastrointestinal system, lower genital system and skin in humans. They cause local or systemic infections and complications frequently in the pyogenic character in patients with suppressed cellular and humoral immunity, such as children, overweight individuals, diabetic patients, and pregnant women. It is known that BHS serogroups and their types responsible for infections are as effective as host-related factors in the prognosis of infections. In this context, tonsillopharyngitis and its complications and skin and soft tissue infections such as erysipelas, impetigo, cellulitis and necrotizing fasciitis are mostly associated with Group A Betahemolytic streptococcal infections, while $\mathrm{C}$ and $\mathrm{G}$ groups are associated with more benign upper respiratory tract infections as well as uncomplicated skin-soft tissue infections and gastrointestinal system infections. It has been shown that Streptococcus agalactiae, Streptococcus dysgalactiae subsp. equisimilis (SDSE) and S. anginosus, located in the flora of female genital tract as opportunistic commensals, may lead to mortal invasive and non-invasive infections such as puerperal sepsis, bacteremia, endocarditis, meningitis, arthritis, osteomyelitis, pneumonia, toxic shocklike syndrome, and rhabdomyolysis in mother and neonate after delivery through vaginal delivery [1-5]. Today, the genotypic surveillance of SDSE strains is gradually gaining importance due to the similarity of $S$. pyogenes infections with the infections they cause in terms of the clinical and epidemiological characteristics [6,7].

The most important virulence factor of microorganism that affects the prognosis of infection in pyogenic streptococci is M protein, which plays a role in adhesion and immune system evasion. The M protein encoded in the emm in the chromosome is an antigenic protein found on the cell surface. This protein, which has many serotypes with phase and size variations, is responsible for the evasion of the host immune system by the microorganism, the formation of reinfections and reactivations. On the other hand, it has 
been claimed that emm genotypes might be associated with different clinical manifestations. Thus, a database containing emm homologous sequences has been established in the $\mathrm{CDC}$ data bank among streptococci strains of Group $\mathrm{C} / \mathrm{G}$, like emm polymorphism database used in the surveillance of $S$. pyogenes strains. The DNA sequence analysis method is used in genotypic surveillance studies based on emm polymorphism [8-10].

Streptococcal infections and related sequelae are important health problems in our country as well as all over the world. The detection of $\mathrm{M}$ serotypes, the major virulence factor on the surface of streptococci, and their effects on clinical prognosis would provide the chance of early and rational intervention for infections and complications. There are no studies on the emm types of group $\mathrm{C}$ and $\mathrm{G}$ streptococci in our country. Therefore, information about group $\mathrm{C}$ and $\mathrm{G}$ streptococcal infections and pathogenesis is also limited, and this project is the first study in Turkey.

The aim of this study is to investigate the emm types and their distribution among the GCS/GGS strains isolated from clinical samples.

\section{Material and methods}

This study was carried out with the approval of Çukurova University Faculty of Medicine Non-Interventional Clinical Research Ethics Committee (Date: 01.04.2016 and Decision No: 2016/19). 98 BHS isolates isolated from different clinical samples in Çukurova University/Balcalı Hospital and identified with Vitek 2GP-ID system were included in the study between November 2015 and January 2017. The Streptococcal Grouping Kit (Oxoid) was used for the serotypic identification of these isolates. The $S$. anginosus strains were genotypically evaluated by the PCR method with EL-F (5'ACTCTTGTGTTAAATAAAATCC-3') and EL-R (5'ACGCAGCATTTTGAAGRGCA-3') primers based on groESL sequences [11]. The PCR conditions were performed in 30 cycles, each cycle being 1 minute at $94{ }^{\circ} \mathrm{C}, 30$ seconds at $53^{\circ} \mathrm{C}$ and 1 minute at $72^{\circ} \mathrm{C}$ and then 7 minutes at 72 ${ }^{\circ} \mathrm{C}$. The PCR products were electrophoresed on $1.5 \%$ agarose gel, stained with ethidium bromide and photographed under UV light.

The clinical isolates were evaluated by emm type-specific PCR and Sequence analysis methods in respect of genotypic characteristics. For the detection of $\mathrm{M}$ protein in streptococci and replication of the emm region, the protocol that the $\mathrm{CDC}$ applied was taken into consideration and the CDC database was used for emm typing [9].

\section{Results}

In the study, 67 isolates were found to be included in $\mathrm{C} / \mathrm{G}$ groups in the serotypic identification of 98 BHS isolates with the Streptococcal Grouping Kit (Oxoid). Of these strains, a total of 67 isolates including SDSE (56), S. anginosus (8), S. equi subsp. zooepedimicus (3), identified phenotypically by Vitek 2GP ID system, were included in the study. SDSE (56) and S. anginosus (8) isolates were confirmed by the PCR method with primers based on groESL sequences (Figure 1).

The determination and prevelance of emm types of a total of 67 isolates, which were SDSE (56), S. equi subsp. zooepedimicus (3) and S. anginosus (8), were examined. As a result of emmPCR carried out for this purpose, in which emmSeq primer sequences were used, the emm gene was detected in 54 of 56 SDSE isolates, while presence of no emm gene was observed in 2 isolates. The emm gene was detected in only 2 of $S$. equi subsp. phenotypically confirmed $S$. dysgalactiae subsp. equisimilis and

Figure 1 - SDSE (361bp) and S. anginosus (742 bp) isolates gel electrophoresis of groESL-PCR products.
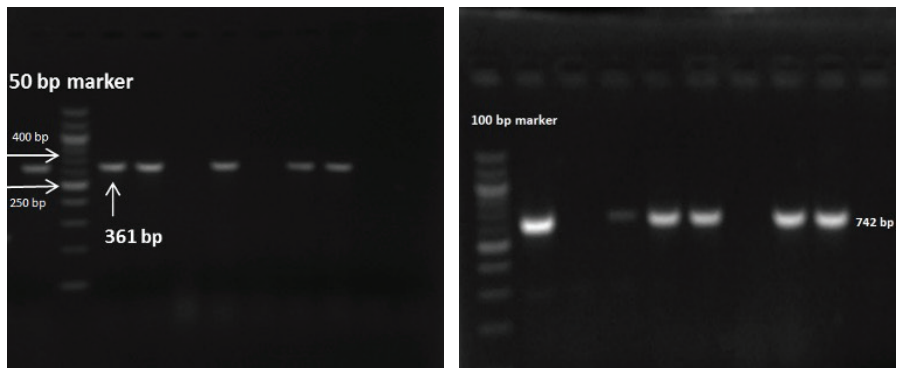

zooepedimicus isolates. However, no emm gene was detected in $8 \mathrm{~S}$. anginosus isolates, either (Table 1).

54 SDSE isolates and 2 S.equi subsp.zooepedimicus, $\mathrm{emm}$ gene of which was detected in consequence of emm-PCR, were evaluated for emm typing. When the patient material from

Table 1
$\begin{aligned} & \text { Detection of the emm gene in SDSE and S. } \\
& \text { anginosus isolates. }\end{aligned}$
\begin{tabular}{|l|l|l|l|}
\hline Type & Number & emm-PCR (+) & emm-PCR (-) \\
\hline SDSE & 56 & 54 & 2 \\
\hline S. anginosus & 8 & 0 & 8 \\
\hline S. equi subsp zooepedimicus & 3 & 2 & 1 \\
\hline
\end{tabular}

which SDSE strains taken under evaluation were isolated was examined, it was seen that the strains were isolated from the blood samples (31) the most and from the abscess material (3) the least (Table 2).

Table 2

Distribution of SDSE samples according to the patient material.

\begin{tabular}{|l|l|l|l|l|l|}
\hline Strain no & Material & Strain no & Material & Strain no & Material \\
\hline S1 & Blood & S19 & Throat & S37 & Throat \\
\hline S2 & Throat & S20 & Blood & S38 & Blood \\
\hline S3 & Blood & S21 & Blood & S39 & Blood \\
\hline S4 & Abscess & S22 & Abscess & S40 & Blood \\
\hline S5 & Blood & S23 & Throat & S41 & Blood \\
\hline S6 & Throat & S24 & Absces & S42 & Throat \\
\hline S7 & Blood & S25 & Blood & S43 & Blood \\
\hline S8 & Blood & S26 & Blood & S44 & Blood \\
\hline S9 & Throat & S27 & Throat & S45 & Throat \\
\hline S10 & Blood & S28 & Wound & S46 & Blood \\
\hline S11 & Throat & S29 & Wound & S47 & Blood \\
\hline S12 & Blood & S30 & Blood & S48 & Blood \\
\hline S13 & Throat & S31 & Blood & S49 & Throat \\
\hline S14 & Wound & S32 & Wound & S50 & Blood \\
\hline S15 & Blood & S33 & Blood & S51 & Blood \\
\hline S16 & Throat & S34 & Blood & S52 & Blood \\
\hline S17 & Blood & S35 & Throat & S53 & Blood \\
\hline S18 & Blood & S36 & Wound & S54 & Blood \\
\hline
\end{tabular}

Table 3 $\mathbf{e m m}$ types detected in SDSE isolates.

\begin{tabular}{|l|l|l|}
\hline emm type & Number (54 isolates) & Percent (\%) \\
\hline stG485 & 28 & 51.85 \\
\hline stG643 & 7 & 12.96 \\
\hline stG6 & 5 & 9.25 \\
\hline stG480 & 4 & 7.40 \\
\hline stG840 & 4 & 7.40 \\
\hline stG6792 & 3 & 5.55 \\
\hline stG6.1 & 2 & 3.70 \\
\hline stG4222 & 1 & 1.85 \\
\hline
\end{tabular}


In the emm typing of SDSE isolates using the CDC database, 8 different emm types were identified, and it was found that the most frequent types were stG485 (\%51.85), stG643 (\%12.96), stG6 (\%9.25), respectively, and the least frequent type was $s t G 4222(\% 1.85)$. Additionally, stG6792 type was identified in 2 S.equi subsp.zooepedimicus isolates (Table 3 ).
When the distribution of the detected emm types according to the patient material was examined, it was determined that stG485 type was most frequently isolated from blood material and stG643 type was most frequently isolated from throat material. The distribution of other SDSE strains according to their emm and material types is given in the table below (Table 4).

\begin{tabular}{|l|l|l|l|l|l|l|l|l|}
\hline Strain no & Material & emm type & Strain no & Material & emm type & Strain no & Material & emm type \\
\hline S1 & Blood & stG485 & S19 & Throat & stG485 & S37 & Throat & stG643 \\
\hline S2 & Throat & stG643 & S20 & Blood & stG6 & S38 & Blood & StG485 \\
\hline S3 & Blood & stG6 & S21 & Blood & stG485 & S39 & Blood & StG485 \\
\hline S4 & Abscess & stG480 & S22 & Abscess & stG485 & S40 & Blood & stG643 \\
\hline S5 & Blood & StG485 & S23 & Throat & stG643 & S41 & Blood & stG480 \\
\hline S6 & Throat & stG840 & S24 & Abscess & stG4222 & S42 & Throat & StG485 \\
\hline S7 & Blood & stG485 & S25 & Blood & stG485 & S43 & Blood & stG840 \\
\hline S8 & Blood & stG485 & S26 & Blood & stG485 & S44 & Blood & stG6.1 \\
\hline S9 & Throat & stG6792 & S27 & Throat & stG643 & S45 & Throat & stG643 \\
\hline S10 & Blood & stG6792 & S28 & Wound & stG480 & S46 & Blood & StG485 \\
\hline S11 & Throat & StG485 & S29 & Wound & stG840 & S47 & Blood & StG485 \\
\hline S12 & Blood & stG840 & S30 & Blood & StG485 & S48 & Blood & StG485 \\
\hline S13 & Throat & stG485 & S31 & Blood & stG6792 & S49 & Throat & StG485 \\
\hline S14 & Wound & stG6 & S32 & Wound & stG6.1 & S50 & Blood & StG485 \\
\hline S15 & Blood & stG485 & S33 & Blood & StG485 & S51 & Blood & StG485 \\
\hline S16 & Throat & stG6 & S34 & Blood & StG485 & S52 & Blood & StG485 \\
\hline S17 & Blood & stG485 & S35 & Throat & stG643 & S53 & Blood & StG485 \\
\hline S18 & Blood & stG6 & S36 & Wound & stG480 & S54 & Blood & StG485 \\
\hline
\end{tabular}

\section{Discussion}

BHSs are a temporary part of normal flora in humans, and recent studies have reported that they increasingly may lead to various invasive and non-invasive infections such as pharyngitis, cellulitis, sepsis, meningitis, endocarditis, acute rheumatic fever, and poststreptococcal glomerulonephritis. GCS/GGS-related infections have been associated with predisposing factors such as bacteremia, alcoholism, diabetes mellitus, malignancy, intravenous substance use, or rupture of the skin. It has been carefully emphasized in recent studies that the major factor in BHS infections is the M protein. However, in many regions of the world, regarding GAS infections, importance is attached to the detection, prevalence, surveillance and epidemiological studies of emm type, while there is not a sufficient number of studies about GCS/GGS. Currently, the global burden of disease caused by SDSE is still not known in many parts of the world because it is not a common practice to identify Group C and G streptococci at the species level in clinical laboratories [12].

Although the number of studies on GAS emm typing is limited in our country, there is no study regarding the emm typing of GCS/GGS. In this study, our aim was the emm detection of a total of 67 isolates including $S$. dysgalactiae subsp. equisimilis (56), S. anginosus (8) and S. equi subsp. zooepedimicus (3), isolated from the patients presented with various clinical complaints to clinics/outpatient clinics during a period of approximately 14 months. As a result of emm-PCR carried out for this purpose, no presence of emm in 2 of 56 SDSE isolates, 1 of $3 S$. equi subsp. zooepedimicus isolates and $8 S$. anginosus isolates was detected, and only 56 SDSE isolates and 1 S. equi subsp. zooepedimicus isolate were included in the study. Similarly, Reibmann S. et al. determined in their study published in 2010 that 2 of 254 SDSE isolates and all of $59 S$. anginosus isolates had negative emm-PCR result [13]. In their study, Rantala S. et al. also stated that emm was negative in 3 out of 140 SDSE isolates [10].

In our study, 8 different emm types were identified, and it was observed that stG485 (51.85\%), stG643 (12.96\%), stG6 (9.25\%) were the most common types, respectively, while stG4222 $(1.85 \%)$ was the least common type. In addition, stG6792 (2/3) type was determined in S. equi subsp. zooepedimicus isolate. In their study, Kittang B. R. et al. found that stG485, stG643 and stG6 types were more prevalent in the emm typing of 76 SDSE isolates isolated from invasive and non-invasive infections [14].

Poradosu et al. reported that 13 different emm types were present in the sequence typing of 56 GGS isolates and that the predominant type was stG48512. When Tseng et al. in Taiwan examined the distribution of emm types in 274 SDSE isolates, they reported that stG485 (45/274) was the most common type, followed by stG6.1 (43/274), stC839 (32/274), stG652 (24/274) and stG652.1 (17/274) types, which they reported to be common [15].

In their study carried out in South India, Reibmann et al. identified 44 different emm types in 252 SDSE isolates and reported that the most common types were stG245 (32), stG6792 (18), stG643 (17), stG6 (14), respectively [13].

When we compared the emm types detected in our study with those of other studies, we observed that they were similar to the emm types seen in different regions. Furthermore, in our study, upon examining the distribution of emm types according to the patient material, we identified stG485 type most frequently in SDSE isolates isolated from blood samples. In blood samples taken from patients with septicemia, Rantala et al., and in SDSE isolates isolated from the blood culture obtained from various 
clinics, Poradosu et al. found that the most common type was stG485, and the most common type was determined to be stG6.1 and the second most common type was determined to be stG485 by Tseng et al. in blood samples in their study conducted in Taiwan [10-12-15].

In our study, the second most common stG643 emm type was detected in SDSE isolates isolated from the throat material. Anand et al. determined that stG643 type was predominant in SDSE isolates isolated from the throat material [16].

Consequently, identification of the GCS/GGS strain will enrich epidemiological data. Additionally, the definition of GCS/GGS-associated infections is important for the treatment of patients. We think that the relationship between emm types and GCS / GGS infections should be examined with future studies. In addition, this study will guide researchers in phenotypicgenotypic identification in future studies with Non-Group A Beta-Hemolytic Streptococci.

This study has limitations. Types of emm and their relationship with infection should also be examined. Examining the relationship between emm types and infection in streptococci will contribute to epidemiological studies.

\section{Conclusion}

As a result, today, GGS and GSS, which cause invasive and non-invasive infections, are still not treated seriously across the world, and there is no sufficient number of studies on the infection load, virulence factors, epidemiological and surveillance studies of these microorganisms. In the near future, these ignored microorganisms will probably increase in patients with chronic disease and many predisposing factors.

Specifically, the identification of emm types remains important for identifying the incidence, relation with infections and rapid changes in species distribution, which may lead to an increase in mortality. In this context, further studies on the relationship between emm types and infections should be demanded to be done. We think that this study, which is the first in our country, will be a reference for other studies to be conducted and the emm types should be examined with studies attended by multicentre and large groups related to GGS/GSS that cause serious infections.

Authors' Contributions: SK collected samples, cultured the isolates, and performed DNA extraction, PCR, emm sequencing. FK and SK designed the study. FK and SK supervised the practical work and data management. SK, CÖG and FK wrote the manuscript. All authors approved the final version of the manuscript.

Ethics approval and consent to participate: This study was carried out with the approval of Çukurova University Faculty of Medicine Non-Interventional Clinical Research Ethics Committee (Date: 01.04.2016 and Decision No: 2016/19).

Disclosures: There is no conflict of interest for all authors.

Acknowledgement: We would like to acknowledge Çukurova University for financially supported. We would like to acknowledge Prof. Dr. Androulla Efstratiou and Dr. Victoria Jane Chalker for their help in the implementation of the emm protocol.

Funding: This study was financially supported by Cukurova University Scientific Research Projects (TDK2015-4861), Adana, Turkey. The funding body supported the laboratory work. The funding body had no contribution in study design, data analysis, interpretation and in writing of the manuscript.

\section{References}

1. Erayman İ, Arıbaş ET, Yılmaz A, Bitirgen M. Boğaz Kültürlerinden İzole Edilen Beta Hemolitik Streptokokların Serogrupları. Türk Mikrobiyol Cem Derg. 2000; 30:13-16.

2. Stevens DL, Kaplan EL. Streptococcal infections: clinical aspects, microbiology, and molecular pathogenesis. New York, NY: Oxford University Press. 2000. [Google Scholar].

3. Musser JM, Shelburne SA. A decade of molecular pathogenomic analysis of group A Streptococcus. J Clin Invest. 2009; 119: 24552463. https://doi:10.1172 / JCI38095.

4. Vandamme P, Pot B, Falsen E, Kersters K, Devriese LA. Taxonomic study of Lancefield streptococcal groups C, G, and L (Streptococcus dysgalactiae) and proposal of S. dysgalactiae subsp. equisimilis subsp. nov. Int J Syst Bacterio.l 1996; 46:774-781. https:// doi:10.1099/00207713-46-3-774.

5. Ruoff KL Streptococcus anginosus (“Streptococcus milleri”): the unrecognized pathogen. Clin Microbiol Rev.1988; 102-108. https:// doi:10.1128/cmr.1.1.102.

6. Kittang, B. R., Bruun, T., Langeland, N., Mylvaganam, H., Glambek, M. \& Skrede, S. Invasive group A, C and G streptococcal disease in western Norway: virulence gene profiles, clinical features and outcomes. Clin Microbiol Infect. 2011; 17:358-364. https://doi. org/10.1111/j.1469-0691.2010.03253.x.

7. Collins CM, Kimura A, Bisno AL. Group G streptococcal M protein exhibits structural features analogous to those of class I M protein of group A streptococci. Infect Immun. 1992; 60:3689-96. https://doi:10.1128/IAI.60.9.3689-3696.1992.

8. Olive C, Schulze K, Sun HK. Enhanced protection against Streptococcus pyogenes infection by intranasal vaccination with a dual antigen component M protein/SfbI lipid core peptide vaccine formulation. Vaccine. 2007; 25:1789-1797. https://oi:10.1016 / j.vaccine.2006.11.031.

9. CDC (Centers for Disease and Prevention). (https://www2a.cdc.gov/ncidod/biotech/strepblast.asp).

10. Rantala S, Vähäkuopus S, Varkila JV, Vuento R, and Syrjänen J. Streptococcus dysgalactiae subsp. Equisimilis Bacteremia, Finland, 1995-2004. Emerging Infectious Diseases. 2010; 16:5. https://doi:10.3201/eid1605.080803.

11. Liu LC, Tsai JC, Hsueh PR and Lee-Jene Teng LJ. Rapid Differentiation between Members of the Anginosus Group and Streptococcus dysgalactiae subsp. equisimilis within Beta-Hemolytic Group C and G Streptococci by PCR. Journal of Clinical Microbiology. 2006; 1836-1838. https:// doi:10.1128/jcm.44.5.1836-1838.2006. 
12. Poradosu RC, Jaffe J, Lavi D, Greenzaid SG, Paz RN, Valinsky L, et al. Group G Streptococcal Bacteremia in Jerusalem. Emerging Infectious Diseases. 2004; 10: 1455-1460. https://doi:10.3201/eid1008.030840

13. Reissmann S, Friedrichs C, Rajkumari R, Itzek A, Fulde M, Rodloff AC, et al. Contribution of Streptococcus anginosus to infections caused by groups C and G streptococci, Southern India. Emerg Infect Dis. 2010; 16:656-663. https://doi:10.3201/eid1604.090448.

14. Kittang BR, Skrede S, Langeland N, Haanshuus CG, Mylvaganam H. emm gene diversity, superantigen gene profiles and presence of SlaA among clinical isolates of group A, C and G streptococci from western Norway. Eur J Clin Microbiol Infect Dis. 2011; 30:423-433. https://doi:10.1007/s10096-010-1105-X.

15. Tseng SP, Lin YY, Tsai JC, Hsueh PR, Chen HJ, Hung WC, Teng LJ. Distribution of emm Types and Genetic Characterization of the mgc Locus in Group G Streptococcus dysgalactiae subsp. equisimilis from a Hospital in Northern Taiwan. Journal of Clinical Microbiology 2010; 2975-2977. https://doi:10.1128/jcm.00444-10.

16. Anand TD, Rajesh T, Rajendhran J, Gunasekaran P. Superantigen profiles of emm and emm-like typeable and nontypeable pharyngeal streptococcal isolates of South India. Annals of Clinical Microbiology and Antimicrobials. 2012; 11:3. https://doi:10.1186/1476-071111-3. 